\title{
Endoscopic Submucosal Dissection for Neoplasia of the Greater Curvature of the Upper and Middle Stomach: J-shaped Superficial Cutting and Splashed Dissection
}

\author{
Noriko Nishiyama ${ }^{1}$, Hideki Kobara ${ }^{1}$, Shintaro Fujihara ${ }^{1}$, Kazuhiro Koduka ${ }^{1}$, Taiga Chiyo ${ }^{1}$, Nobuya Kobayashi ${ }^{1}$, Kouji \\ Fujita $^{1}$, Maki Ayaki ${ }^{1}$, Tatsuo Yachida ${ }^{1}$, Joji Tani ${ }^{1}$, Tingting Shi ${ }^{1}$, Keiichi Okano ${ }^{2}$, Yasuyuki Suzuki ${ }^{2}$, Hirohito Mori ${ }^{1}$, \\ Tsutomu Masaki ${ }^{1}$
}

1) Gastroenterology and

Neurology, Faculty of

Medicine, Kagawa University,

Kagawa

2) Gastroenterological Surgery,

Faculty of Medicine, Kagawa

University, Kagawa, Japan

\begin{abstract}
Background \& Aims: Endoscopic submucosal dissection (ESD) has become a standard treatment for early gastric neoplasia. However, as the upper and middle body of the greater curvature has a rich vasculature and submucosal fibrosis, ESD of neoplasia in these locations requires a specific strategy. We aimed to investigate the efficacy and safety of the J-shaped superficial cutting and splashed submucosal dissection (JSCS) technique for neoplasia of the greater curvature by comparing ESD using JSCS with conventional ESD.

Methods: Twenty-two patients who underwent ESD for gastric neoplasia affecting the upper and middle body of the greater curvature were divided into two groups for retrospective analysis. Nine patients underwent conventional ESD (c-Group), while 13 underwent ESD with JSCS (j-Group). Primary outcome was the en bloc resection rate. Secondary outcomes included complete resection (R0) rate, procedure time, perforation rate, total bleeding time, and the total number of massive bleeding events and of hemostatic forceps times applied during ESD.

Results: There were no significant differences between both groups (c-Group vs j-Group) in en bloc resection rate, or R0 resection rate. Compared with the c-Group, the $\mathrm{j}$-Group tended to have a decreased mean procedure time (mean 133 minutes vs 74 minutes, $\mathrm{p}=0.11$ ) and perforation rate $(11 \% \mathrm{vs} 0 \%, \mathrm{p}=0.41)$. Compared with the c-Group, the j-Group had significantly fewer bleeding incidents (13.4 times vs 6.6 times, $\mathrm{p}=0.0095)$, shorter total bleeding time (17.6 min vs $7.4 \mathrm{~min}, \mathrm{p}=0.036$ ), and fewer usages of hemostatic forceps (6.3 times vs 2.4 times, $\mathrm{p}=0.026$ ) during ESD.

Conclusion: Endoscopic submucosal dissection with JSCS is superior to conventional ESD, as it reduces intraprocedural bleeding. This technique has the potential to become the standard strategy for neoplasia affecting the upper and middle body of the greater curvature.
\end{abstract}

Key words: endoscopic submucosal dissection - early gastric cancer - greater curvature.

Abbreviations: ESD: endoscopic submucosal dissection; JSCS: J-shaped superficial cutting and splashed submucosal dissection; R0: complete resection.

\section{INTRODUCTION}

Endoscopic submucosal dissection (ESD) has recently become a common minimally invasive therapy for early gastric neoplasia. The major advantages of ESD are a higher en bloc resection rate and more accurate histologic evaluation than endoscopic mucosal resection [1, 2]. However, the disadvantages of ESD are that complicated cases require a high degree of skill, and that ESD has a greater prevalence of adverse events such as perforation and bleeding than endoscopic mucosal resection. The degree of difficulty of the ESD procedure is influenced by several factors: technical aspects, lesion characteristics such as size and location, circumferential instruments, and therapeutic strategy. Although safer and more reliable devices (such as scissor forceps) have been developed [3], the most important factor affecting the successful completion of ESD is considered to be the therapeutic strategy, including how to resect and where to make the first approach. The technical difficulty of ESD is affected by the visibility of the submucosal layer, which depends on the vasculature and submucosal fibrosis [4].

As the anatomical characteristics of the submucosal layer differ substantially between locations, individual strategies are required. In particular, the greater curvature of the upper and 
middle body of the stomach has a rich vasculature and submucosal fibrosis. Moreover, lesions at these locations are submerged due to gravity. Hence, the visualization of the submucosal layer is often poor due to frequent bleeding and fibrosis, leading to increased procedural difficulty, increased procedural time, and the occurrence of adverse events in clinical practice [5]. Thus, a specific strategy is needed to perform effective and safe ESD for neoplasia located in the greater curvature of the upper and middle body of the stomach. We found that the J-shaped superficial cutting and splashed submucosal dissection (JSCS) technique is effective for ESD of the greater curvature.

The present study aimed to investigate the efficacy and safety of the JSCS technique by comparing ESD using JSCS with conventional ESD without JSCS in patients with gastric neoplasia located in the greater curvature of the upper and middle stomach.

\section{METHODS}

\section{Patients}

The present study included 54 patients who underwent ESD for gastric neoplasia affecting the greater curvature between June 2011 and August 2017 at Kagawa University Hospital. While we performed “conventional ESD” between June 2011 and March 2014, we performed a newly developed "ESD using JSCS” in order to improve technical aspects between April 2014 and August 2017. Thus, the criterion to choose the procedure was based on two different periods of time.

Gastric neoplasia included early gastric cancer and adenoma that could not be preoperatively distinguished from early gastric cancer. ESD was indicated for early gastric cancer in which the invasion depth was expected to be less than slightly submucosal $(500 \mu \mathrm{m})$ and there was no possible lymph node metastasis, based on Japanese ESD criteria. The inclusion criterion was a lesion affecting the greater curvature of the upper and middle body of the stomach, as this location has proved to be difficult to perform gastric ESD due to local anatomical features. Twenty-eight patients were excluded from the study, as the lesions were located in the lower body of the stomach (the lesion does not submerge). One patient was excluded as the lesion was in the remnant stomach and three patients were excluded as the lesions were accompanied by ulcerations. A lesion with an ulcer is commonly considered difficult for ESD, the successful ESD depending on the degree of submucosal fibrosis and the skill level of the performers.

In the end, 22 patients who underwent ESD for early gastric neoplasia affecting the upper and middle body of the greater curvature were included. The patients were divided into two groups for retrospective analysis: 9 cases of conventional ESD (c-Group), and 13 cases of ESD with the JSCS technique (j-Group). The flowchart for the two groups is shown in Fig 1.

The Clinical Ethics Committee of Kagawa University Hospital approved this study (Clinical Study registration number: UMIN000032713). All the patients provided written informed consent to undergo the procedures and to participate in the study.

\section{Outcome measures}

The primary outcome measure was the en bloc resection rate. Secondary measures were complete resection rate, procedure time, perforation rate, the total number of massive bleeding events, total bleeding time and the number of times that hemostatic forceps were used during ESD. Total numbers of massive bleeding events, total bleeding time and hemostatic forceps usages during ESD were calculated by reviewing the video recordings of the ESD procedures.

\section{Definitions}

En bloc resection was defined as the macroscopic removal of the whole tumor specimen in one piece. Complete resection (R0) rate was defined as curative resection with negative margins, and the absence of lymph node and vascular invasion (confirmed by histological assessment of the resected specimen). The procedure time was the interval from precutting to the completion of specimen resection. A diagnosis of

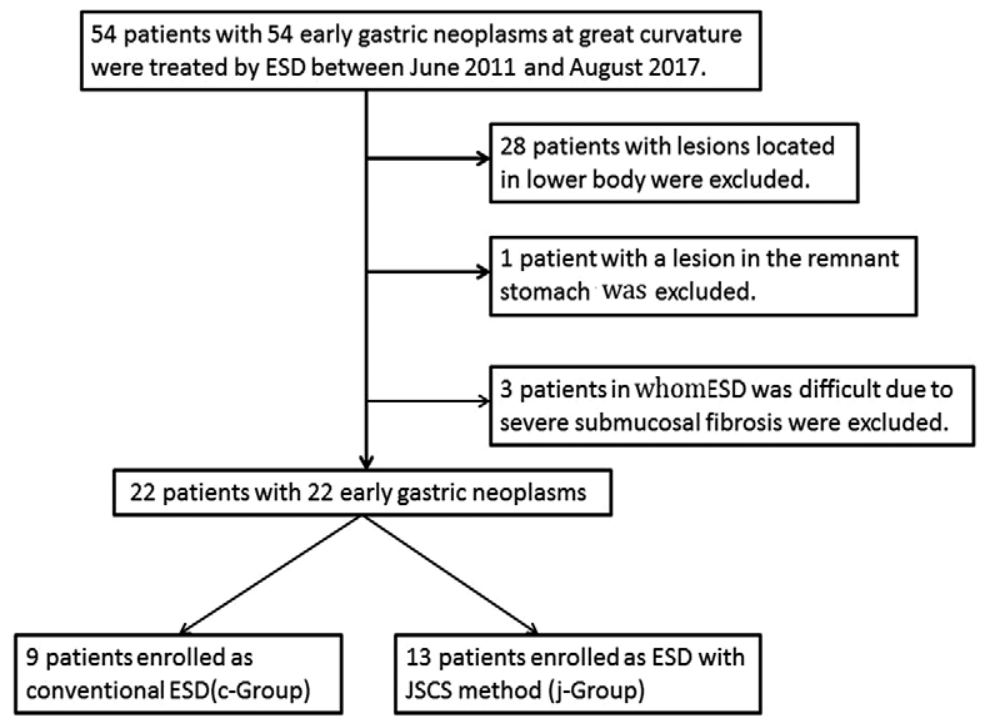

Fig. 1. Flowchart of the patients enrolled in this study and their lesions. ESD: endoscopic submucosal dissection; JSCS: J-shaped superficial cutting and splashed submucosal dissection. 
perforation was confirmed by the appearance of the extramural organ or greater omentum during ESD. A massive bleeding event was considered as bleeding that continued for more than 5 seconds during ESD (from pre-cutting to completion of specimen resection). The total number of times that hemostatic forceps were used during ESD was defined as the total number of times that hemostasis was achieved using hemostatic forceps during ESD (from pre-cutting to completion of specimen resection).

\section{Basic ESD procedure}

All patients were treated under deep sedation with pentazocine and midazolam or propofol. ESD was carried out using a single-channel video endoscope (GIF-Q260J or GIFH260Z; Olympus, Tokyo, Japan). Carbon dioxide insufflation was used during the ESD procedure. Transparent caps (Elastic Touch F-010 or F-030, Top Corporation, Tokyo, Japan) were attached to the endoscopic tip. The electrosurgical unit was a VIO 300D (ERBE, Elektromedizin, Tübingen, Germany). Marking dots were circumferentially placed approximately 5 $\mathrm{mm}$ outside of the lesion using an electrosurgical Dual knife (KD-441Q; Olympus, Tokyo, Japan) with forced coagulation mode (VIO300D; effect 4,40W). A 1:1 solution of $0.4 \%$ hyaluronate sodium (MucoUp; Johnson and Johnson K.K., Tokyo, Japan) and glycerol (Chugai Pharmaceutical Co. Ltd, Tokyo, Japan), mixed with diluted epinephrine (1:200,000) and indigo carmine, was injected into the submucosal layer using a 25-G needle (Boston Scientific, Tokyo, Japan). Circumferential incision of the mucosa and submucosal dissection was achieved using a Dual knife or an IT Knife-2 (Olympus) with the EndoCut mode (effect 2, duration 3) or the Swift mode (effect $4,60 \mathrm{~W}$ ) provided by the VIO300D, respectively. Bleeding was managed in all procedures using hemostatic forceps (FD-410
LR; Olympus) with the soft coagulation mode (VIO300D; effect $6,80 \mathrm{~W}$ ). The approach for all procedures was toward the targeted lesions under retroflex view.

All procedures were performed by four endoscopists (N.N., H.M., H.K., and T.Y.) who had successfully experienced more than 200 gastric ESD cases.

\section{Conventional ESD technique (c-Group)}

The schema of the conventional endoscopic submucosal dissection procedure is summarized in Fig. 2.

Pre-cutting was performed at the oral side using the Dual knife, and then the mucosal incision was performed at the greater curvature side with the IT Knife- 2 from the oral precutting side to the anal side. Next, the trimming of the mucosal cutting site was performed by dissecting sufficiently deeply into the submucosa until the muscularis propria appeared. After residual circumferential mucosal cutting and trimming from the anal side to the oral side, the residual submucosa was dissected by entering the submucosal space.

J-shaped superficial cutting and splashed submucosal dissection technique (j-Group)

The schema of the JSCS procedure is summarized in Fig. 3.

A superficial incision line in the mucosal epithelium above the submucosa was created at the greater curvature side from the oral side to the anal side using the Dual knife, like drawing a J-shaped curve (Fig. 4a). After a submucosal space was made at the anal side, the submucosa below the superficial incision line made at the greater curvature side was dissected by splashing the IT Knife-2 toward the intralumen from the anal side to the oral side while visually identifying the submucosal layer (Fig. 4b). Large-sized vessels in the submucosa were cauterized using hemostatic forceps to stop massive bleeding from the

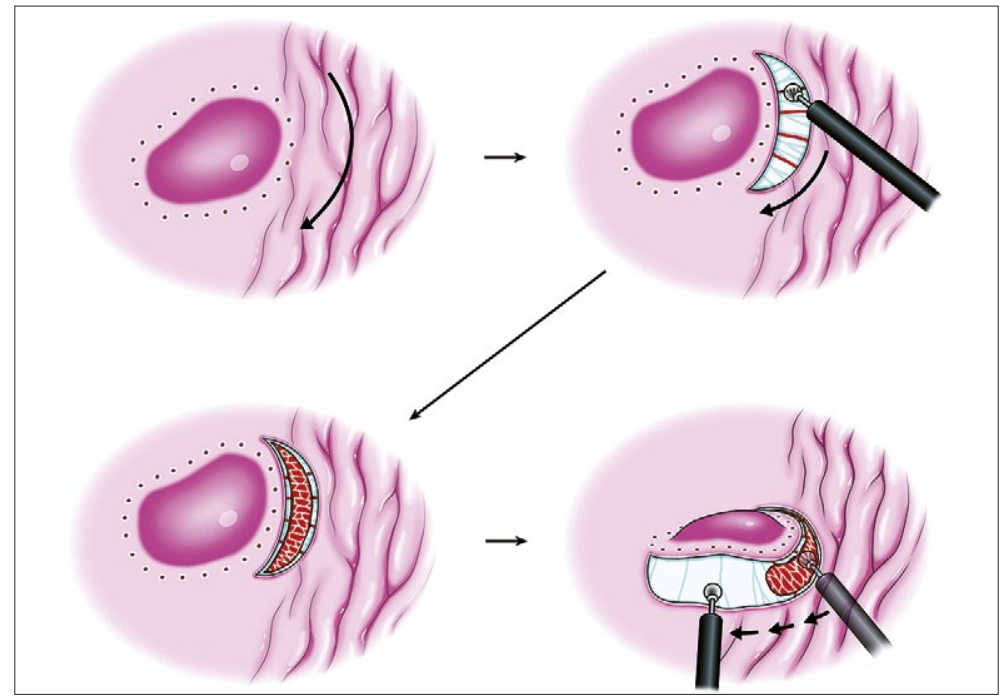

Fig. 2. Schemas showing the conventional ESD procedure. Pre-cutting was performed at the oral side using the Dual knife, and then the mucosal incision was performed at the greater curvature side with the IT Knife- 2 from the oral precutting site to the anal side. Next, the trimming of the mucosal cutting site was performed by dissecting sufficiently deeply into the submucosa until the muscularis propria appeared. After residual circumferential mucosal cutting and trimming from the anal side to the oral side, the residual submucosa was dissected by entering the submucosal space. 


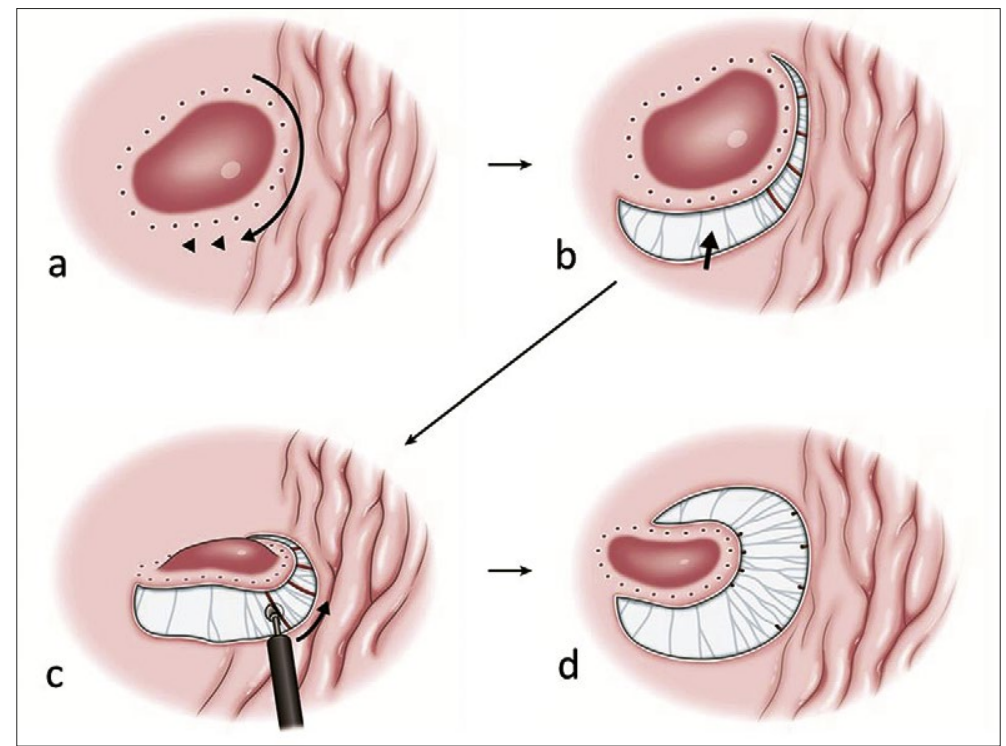

Fig. 3. Schemas showing the JSCS procedure. a. Superficial cutting at the greater curvature (J-shaped curve); b. Entry into the submucosa from anal side; c. Submucosal dissection by splashing an IT Knife-2 toward the intralumen (splashed submucosal dissection); d. Completion of safe and reliable submucosal dissection at the greater curvature side.

greater curvature side (Fig. 4c). After sufficient submucosal dissection at the greater curvature side was performed (Fig. 4d), the residual circumferential mucosal cutting and submucosal dissection formally proceeded from the anal side to the oral side.

\section{Statistical analysis}

Continuous variables were presented as mean value \pm standard deviation. Differences in categorical variables between two groups were examined by Fisher's exact test when

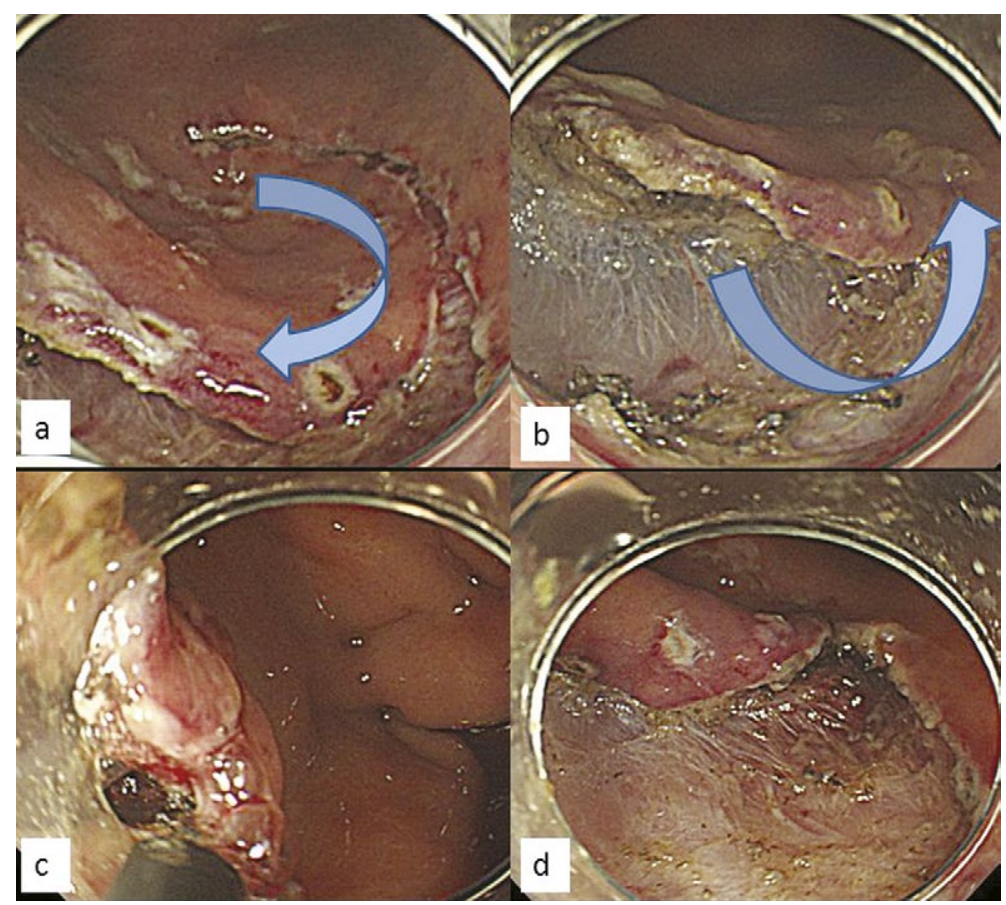

Fig. 4. Pictures illustrating the JSCS procedure. a. A Dual knife was used to create a superficial incision line in the mucosal epithelium above the submucosa at the greater curvature from the oral side to the anal side, like drawing a J-shaped curve (blue arrow); b. A submucosal space was made at the anal side and then submucosal dissection at the greater curvature was attempted (blue arrow); c. Next, the submucosa below the superficial incision line made at the greater curvature side was dissected by splashing an IT Knife- 2 toward the intralumen from the anal side to the oral side while visually identifying the submucosal layer; d. Sufficient submucosal dissection was performed at the greater curvature side. 
required. Continuous variables were compared using Welch's t-test. A p-value $<0.05$ was considered statistically significant. All statistical analyses were conducted using JMP 11.2.0 (SAS Institute Inc., Cary, NC, USA).

\section{RESULTS}

The baseline characteristics of the patients and lesions are shown in Table I. There was no significant difference between the two groups regarding tumor size or location.

No significant differences were observed between the two groups in an en bloc resection rate or $\mathrm{R} 0$ resection rate (Table II). Compared with the c-Group, the j-Group tended to have a decreased procedure time and perforation rate; furthermore, the $\mathrm{j}$-Group had significantly fewer massive bleeding events $(p=0.0095)$, shorter total bleeding time $(p=0.036)$, and the hemostatic forceps were used fewer times $(\mathrm{p}=0.026)$ during ESD (Table II).

No complications such as bleeding and perforation after ESD occurred in either group. In one case, a procedureassociated perforation occurred (Fig. 5), and one representative case with massive bleeding in the c-Group is shown in Figs. $6 \mathrm{a}$ and $6 \mathrm{~b}$.

We monitored all the 22 patients for 1 year.
Three patients (one from the c-Group and two from the j-Group) underwent gastrectomy because of SM massive invasion in tumor depth. No complications and recurrences occurred in all patients during the 1 year follow-up period.

\section{DISCUSSION}

This is the first report to compare the efficacy and safety of a modified ESD using the novel JSCS technique with conventional ESD for gastric neoplasia affecting the upper and middle body of the greater curvature, which is known to be a difficult lesion to treat via gastric ESD. The present results revealed that the JSCS technique might significantly reduce the number of massive bleeding events and the number of situations when hemostatic forceps are used, leading to the achievement of an effective and safe ESD.

Although gastric ESD is widely accepted as an effective treatment, the ESD technique is complicated, requires much experience, and can result in adverse events such as perforation and bleeding [3]. Previous studies reported that the factors related to technical difficulty and long procedure time for gastric ESD were tumor diameter larger than $20 \mathrm{~mm}$, lesions located in the middle or upper third of the stomach or in the greater curvature, and the presence of ulceration or fibrosis [6-13].

Table I. The baseline characteristics of the 22 patients and 22 lesions.

\begin{tabular}{|c|c|c|c|c|}
\hline & Total $(n=22)$ & j-Group $(n=13){ }^{\#}$ & c-Group $(\mathrm{n}=9)^{\# \#}$ & $\mathrm{p}$ value \\
\hline \multicolumn{5}{|l|}{ Patients characteristics } \\
\hline Age $(\text { year })^{*}$ & $73.5 \pm 9.6$ & $73 \pm 9.5$ & $74.4 \pm 10.2$ & $0.74^{(1)}$ \\
\hline Gender, n (\%) & & & & $0.41^{(2)}$ \\
\hline Male & $21(95.4 \%)$ & $13(100 \%)$ & $8(88.8 \%)$ & \\
\hline Female & $1(4.5 \%)$ & $0(0 \%)$ & $1(11.1 \%)$ & \\
\hline \multicolumn{5}{|l|}{ Lesion characteristics } \\
\hline Location, n (\%) & & & & $1.0^{(2)}$ \\
\hline upper body & $8(36.3 \%)$ & $5(38.4 \%)$ & $3(33.3 \%)$ & \\
\hline middle body & $14(63.6 \%)$ & $8(61.5 \%)$ & $6(66.6 \%)$ & \\
\hline Tumor diameter $(\mathrm{mm})^{*}$ & $15.7 \pm 8.6$ & $17.0 \pm 8.7$ & $13.8 \pm 8.6$ & $0.41^{(1)}$ \\
\hline Resected specimen diameter $(\mathrm{mm})^{*}$ & $41.9 \pm 11.6$ & $47.2 \pm 12.1$ & $38.2 \pm 10.1$ & $0.088^{(1)}$ \\
\hline Morphology, n (\%) & & & & $0.15^{(2)}$ \\
\hline Protruded & $9(40.9 \%)$ & $7(53.8 \%)$ & $2(22.2 \%)$ & \\
\hline Flat & $2(9.0 \%)$ & $0(0 \%)$ & $2(22.2 \%)$ & \\
\hline Depressed & $11(50 \%)$ & $6(46.1 \%)$ & $5(55.5 \%)$ & \\
\hline Histology, n (\%) & & & & $0.70^{(2)}$ \\
\hline Differentiated adenocarcinoma & $19(86.3 \%)$ & $12(92.3 \%)$ & $7(77.7 \%)$ & \\
\hline Undifferentiated adenocarcinoma & $2(9 \%)$ & $1(7.69 \%)$ & $1(11.1 \%)$ & \\
\hline Adenoma & $1(4.5 \%)$ & $0(0 \%)$ & $1(11.1 \%)$ & \\
\hline Depth, n (\%) & & & & $0.61^{(2)}$ \\
\hline pTla & $18(81.8 \%)$ & $10(76.9 \%)$ & $8(88.8 \%)$ & \\
\hline pT1b & $4(18.1 \%)$ & $3(23.0 \%)$ & $1(11.1 \%)$ & \\
\hline Ulceration, n (\%) & $0(0 \%)$ & $0(0 \%)$ & $0(0 \%)$ & $1.0^{(2)}$ \\
\hline
\end{tabular}

\#J-shaped superficial cutting and splashed submucosal dissection technique; \#\#Conventional endoscopic submucosal dissection technique; ${ }^{*}$ Mean values are expressed as the mean value \pm standard deviation; ${ }^{(1)}$ Welchi`s t- test; ${ }^{(2)}$ Fisher's exact test 
Table II. Treatment outcomes in the conventional ESD group versus the JSCS group.

\begin{tabular}{|c|c|c|c|c|}
\hline Outcomes & Total $(n=22)$ & $\mathrm{j}$-Group $(\mathrm{n}=13)^{\#}$ & c-Group (n=9) \#\# & $p$ value \\
\hline \multicolumn{5}{|l|}{ Procedure time, $\min$} \\
\hline Mean \pm SD & $102.3 \pm 70.1$ & $74.07 \pm 35.1$ & $133.22 \pm 95.6$ & $0.11^{(1)}$ \\
\hline \multicolumn{5}{|l|}{ Total bleeding time, min } \\
\hline Mean \pm SD & $11.6 \pm 9.8$ & $7.5 \pm 5.0$ & $17.7 \pm 12.0$ & $0.036^{(1)}$ \\
\hline \multicolumn{5}{|l|}{$\begin{array}{l}\text { Total number of massive bleeding } \\
\text { events }\end{array}$} \\
\hline Mean $\pm S D$ & $9.5 \pm 5.8$ & $6.7 \pm 3.9$ & $13.4 \pm 5.8$ & $0.00951^{(1)}$ \\
\hline \multicolumn{5}{|l|}{$\begin{array}{l}\text { Total times using hemostatic } \\
\text { forceps }\end{array}$} \\
\hline Mean \pm SD & $4.1 \pm 3.5$ & $2.5 \pm 1.5$ & $6.3 \pm 4.2$ & $0.027^{(1)}$ \\
\hline En bloc resection rate, $\mathrm{n}(\%)$ & $22(100 \%)$ & $13(100 \%)$ & $9(100 \%)$ & $\mathrm{NS}^{(2)}$ \\
\hline Complete resection rate, $\mathrm{n}(\%)$ & $17(77.2 \%)$ & $10(76.9 \%)$ & $7(77.7 \%)$ & $\mathrm{NS}^{(2)}$ \\
\hline Perforation rate, $\mathrm{n}(\%)$ & $0(0 \%)$ & $0(0 \%)$ & $1(11.1 \%)$ & $0.41^{(2)}$ \\
\hline Post-procedure bleeding rate, $\mathrm{n}(\%)$ & $0(0 \%)$ & $0(0 \%)$ & $0(0 \%)$ & $\mathrm{NS}^{(2)}$ \\
\hline
\end{tabular}

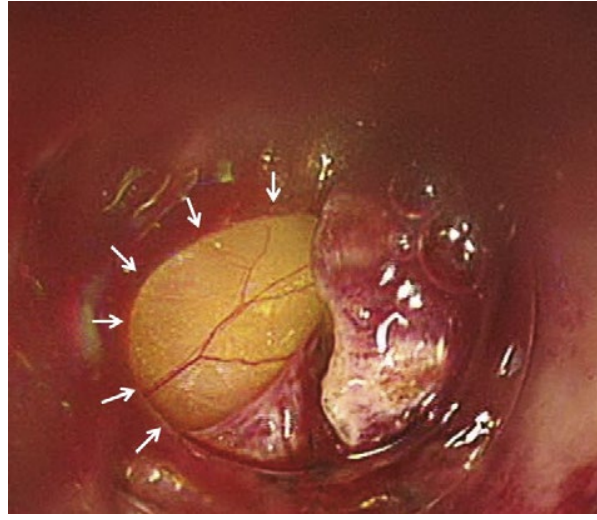

Fig. 5. One case of procedure-associated perforation at the greater curvature side occurred in the c-Group.

The risk of perforation may be affected by the following conditions: thinness of the gastric wall, and presence of severe fibrosis, fatty tissue, and rich vasculature in the submucosal layer [14]. The greater curvature of the upper and middle body of the stomach have anatomical features concordant with the above mentioned conditions, leading to an increased difficulty and decreased safety of ESD in this area [15]. Moreover, as these locations are sites where lesions easily submerge due to gravity, massive bleeding during ESD makes the procedure more difficult. These reasons were considered when the present inclusion criteria were assigned, and when our newly-developed JSCS technique was introduced. One advantage of the JSCS technique is minimization of the risk of injury of the large submucosal vessels at the greater curvature side, through the creation of the superficial mucosal incision line (Fig. 7), and visible large submucosal vessels from the anal side (Fig. 8); in comparison, conventional ESD requires sufficiently deep dissection into the submucosa until the muscle layer is visualized. Another advantage of the JSCS technique is that accidental perforation due to invisible submucosa with fibrosis is prevented by entering the submucosa via the visible
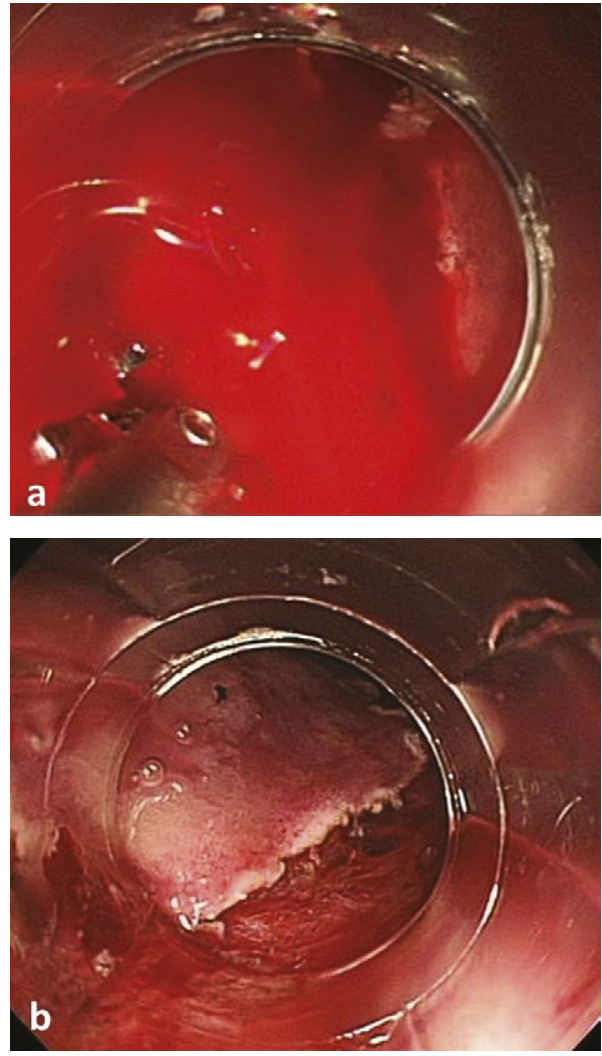

Fig. 6. One representative case with massive bleeding in the c-Group (a). Poor visualization of the submucosal layer occurred due to a submucosal hematoma (b).

submucosal space at the anal side. The submucosa below the superficial incision line made at the greater curvature side can be dissected effectively and safely from the anal side towards oral side, while submucosal vessels can be identified and an accurate distinction can be made between the submucosal layer and the muscle layer. This strategy is an essential key of the JSCS technique. 


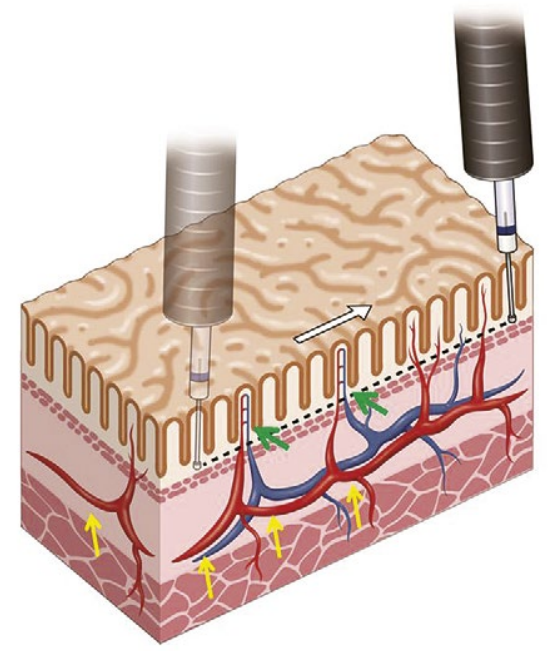

Fig. 7. Schema showing the vessels of the submucosal layer. Large vessels (yellow arrows) exist in the deep submucosal layer, while there are less vessels in the shallow submucosal layer (green arrow).

In the present study, although no significant differences were observed between the two groups in the en bloc resection rate, R0 rate, total procedure time, and perforation rate, the j-Group experienced significantly fewer massive bleeding events and the hemostatic forceps were used significantly fewer times during ESD. This indicates that ESD with JSCS is superior to conventional ESD in terms of reducing the bleeding during the procedure. Future studies involving larger samples may detect significant differences between the two groups in total procedure time and perforation rate. Thus, a prospective comparative study with a large sample size is required to confirm the differences between these methods.

A novel concept designed to make the ESD procedure easier and safer has currently been produced using a counter-traction technique. There are several traction methods being proposed, such as ring-thread counter-traction [16], the dental floss method [17], the double scope method [18, 19], and the robotassisted method [20]. However, these methods are complicated, inconvenient, and carry an increased risk of specimen damage caused by the strong tension that comes from using mechanical devices. Furthermore, one of the most important aspects for effective and safe ESD is to obtain clear visualization, and to

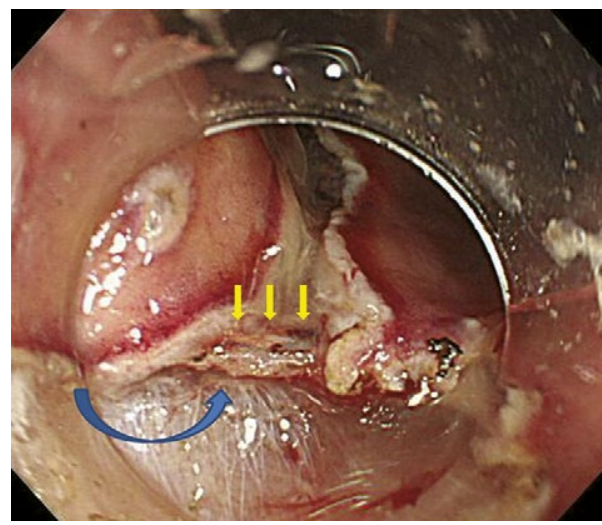

Fig. 8. Minimized injury of large submucosal vessels because of visible large vessels from the anal side. identify the layer and the vessels of the submucosa. From this viewpoint, the JSCS technique and traction methods are both reasonable options. Mori et al. [21] described a novel near-side approach, which applied a step by step incision from the near side to open the incision space quickly and made bleeding control easier [21].

These techniques involving J-shaped superficial cutting and splashed submucosal dissection have become basic techniques for ESD. Thus, if trainees can acquire basic techniques in educational programs, all performers can achieve this procedure. Oyama et al. [22] estimated that the learning curve of ESD would increase through the following educational program. First, trainees have to observe ESD techniques by experts. Second, they should perform at least 15 procedures each in different locations (lower, middle, upper body) of the stomach under expert teaching. When ESD competence (enbloc resection $>80 \%$ and complications $<10 \%$ ) was reached, we considered that ESD using JSCS would be acceptable for trainees.

The strategy of submucosal dissection from anal side to oral side is similar to our strategy. Whereas, our method involves that the incision of the greater curvature side is the first step, and the superficial mucosal incision enables it to minimize the injury of the large submucosal vessels. This technique demonstrates one advantage of our strategy.

Our study has some limitations: small sample size, retrospective analysis and single center study.

\section{CONCLUSION}

This novel JSCS technique is superior to conventional ESD, as it reduces intraprocedural bleeding. This technique has thus the potential to become the standard strategy for neoplasia affecting the upper and middle body of the greater curvature.

\section{Conflicts of interest: None to declare. Funding: None.}

Authors' contributions: H.K. and H.M.: study concept and design; S.F., Y.G., T.C., N.K., T.M., M.A., T.Y.: data acquisition; H.K. and N.N.: data analysis and interpretation; T.M., M.A., N.N.: material supplying; H.K.: statistical analysis. K.O., Y.S., and T.M. supervised the study.

\section{REFERENCES}

1. Gotoda T, Yamamoto H, Soetikno RM. Endoscopic submucosal dissection of early gastric cancer. J Gastroenterol 2006;41:929-942. doi:10.1007/s00535-006-1954-3

2. Isomoto H, Shikuwa S, Yamaguchi N, et al. Endoscopic submucosal dissection for early gastric cancer: a large-scale feasibility study. Gut 2009;58:331-336. doi:10.1136/gut.2008.165381

3. Rösch T, Sarbia M, Schumacher B, et al. Attempted endoscopic en bloc resection of mucosal and submucosal tumors using insulated-tip knives: a pilot series. Endoscopy 2004;36:788-801. doi:10.1055/s-2004-825838

4. Fukami N. What we want for ESD is a second hand! Traction method Gastrointest Endosc 2013;78:274-276. doi:10.1016/j.gie.2013.04.192

5. Oda I, Gotoda T, Hamanaka H, et al. Endoscopic submucosal dissection for early gastric cancer: technical feasibility, operation time and complications from a large consecutive series. Dig Endosc 2005; 17:5458. doi: 10.1111/j.1443-1661.2005.00459.x 
6. Imagawa A, Okada H, Kawahara Y, et al. Endoscopic submucosal dissection for early gastric cancer: results and degrees of technical difficulty as well as success. Endoscopy 2006;38:987-990. doi:10.1055/s-2006-944716

7. Ohta $\mathrm{T}$, Ishihara R, Uedo N, et al. Factors predicting perforation during endoscopic submucosal dissection for gastric cancer. Gastrointest Endosc 2012;75:1159-1165. doi:10.1016/j.gie.2012.02.015

8. Ahn JY, Choi KD, Choi JY, et al. Procedure time of endoscopic submucosal dissection according to the size and location of early gastric cancers: analysis of 916 dissections performed by 4 experts. Gastrointest Endosc 2011;73:911-916. doi:10.1016/j.gie.2010.11.046

9. Jeon SW, Jung MK, Kim SK, et al. Clinical outcomes for perforations during endoscopic submucosal dissection in patients with gastric lesions. Surg Endosc 2010;24:911-916. doi:10.1007/s00464-009-0693-y

10. Abe $\mathrm{Y}$, Inamori $\mathrm{M}$, Iida $\mathrm{H}$, et al. Clinical characteristics of patients with gastric perforation following endoscopic submucosal resection for gastric cancer. Hepatogastroenterology 2009;56:921-914.

11. Kim HJ, Chung H, Jung DH, et al. Clinical outcomes of and management strategy for perforations associated with endoscopic submucosal dissection of an upper gastrointestinal epithelial neoplasm. Surg Endosc 2016;30:5059-5067. doi:10.1007/s00464-016-4854-5

12. Toyokawa $\mathrm{T}$, Inaba $\mathrm{T}$, Omote $\mathrm{S}$, et al. Risk factors for perforation and delayed bleeding associated with endoscopic submucosal dissection for early gastric neoplasms: analysis of 1123 lesions. J Gastroenterol Hepatol 2012;27:907-912. doi:10.1111/j.1440-1746.2011.07039.x

13. Yoo JH, Shin SJ, Lee KM, et al. Risk factors for perforations associated with endoscopic submucosal dissection in gastric lesions: emphasis on perforation type. Surg Endosc 2012;26:2456-2464. doi:10.1007/s00464-012-2211-x

14. Chung IK, Lee JH, Lee SH, et al. Therapeutic outcomes in 1000 cases of endoscopic submucosal dissection for early gastric neoplasms: Korean ESD Study Group multicenter study. Gastrointest Endosc 2009;69:12281235. doi:10.1016/j.gie.2008.09.027
15. Ono S, Kato M, Nakagawa M, Imai A, Yamamoto K, Shimizu Y Outcomes and predictive factors of "not self-completion" in gastric endoscopic submucosal dissection for novice operators. Surg Endosc 2013;27:3577-3583. doi:10.1007/s00464-013-2929-0

16. Mori H, Kobara H, Nishiyama N, Fujihara S, Matsunaga T, Masaki T. Novel effective and repeatedly available ring-thread counter traction for safer colorectal endoscopic submucosal dissection. Surg Endosc 2017;31:3040-3047. doi:10.1007/s00464-016-5326-7

17. Suzuki S, Gotoda T, Kobayashi Y, et al. Usefulness of a traction method using dental floss and a hemoclip for gastric endoscopic submucosal dissection: a propensity score matching analysis (with videos). Gastrointest Endosc 2016;83:337-346. doi:10.1016/j. gie.2015.07.014

18. Higuchi K, Tanabe S, Azuma M, et al. Double-endoscope endoscopic submucosal dissection for the treatment of early gastric cancer accompanied by an ulcer scar (with video). Gastrointest Endosc 2013;78:266-273. doi:10.1016/j.gie.2013.01.010

19. Ahn JY, Choi KD, Choi JY, et al. Transnasal endoscope-assisted endoscopic submucosal dissection for gastric adenoma and early gastric cancer in the pyloric area: a case series. Endoscopy 2011;43:233-235. doi:10.1055/s-0030-1256037

20. Wang Z, Phee SJ, Lomanto D, et al. Endoscopic submucosal dissection of gastric lesions by using a master and slave transluminal endoscopic robot: an animal survival study. Endoscopy 2012;44:690-694. doi:10.1055/s-0032-1309404

21. Mori G, Nonaka S, Oda I, et al. Novel strategy of endoscopic submucosal dissection using an insulation-tipped knife for early gastric cancer: near-side approach method. Endosc Int Open 2015;3:E425-E431. doi:10.1055/s-0034-1392567

22. Oyama T, Yahagi N, Ponchon T, Kiesslich T, Berr F. How to establish endoscopic submucosal dissection in Western countries. World J Gastroenterol 2015;40:11209-11220. doi:10.3748/wjg.v21.i40.11209 